\title{
A Fatal Case of Streptococcal Toxic Shock Syndrome Caused By Genotype Emm1 In The Third Trimester of Pregnancy, China
}

\section{Yasha Luo}

Guangdong Women and Children Hospital

\section{Minling Zheng}

Guangdong Women and Children Hospital

\section{Yanyuan Chen}

Guangdong Women and Children Hospital

\section{Chunming Gu}

Guangdong Women and Children Hospital

\section{Lijuan Lv}

Guangdong Women and Children Hospital

Xiaoping Mu ( $\triangle$ muxiaoping721@163.com )

Guangdong Women and Children Hospital https://orcid.org/0000-0002-5147-9096

\section{Case report}

Keywords: Streptococcus pyogenes, pregnancy, streptococcal toxic shock syndrome, Genotype Emm1, MALDI-TOF, 16S rRNA gene

Posted Date: November 15th, 2021

DOI: https://doi.org/10.21203/rs.3.rs-452042/v2

License: (c) (i) This work is licensed under a Creative Commons Attribution 4.0 International License. Read Full License 


\section{Abstract}

Background: Group A streptococcal (GAS) toxic shock syndrome (TSS) is a rare invasive disease, causing a high risk of maternal and fetal mortality during pregnancy. We report a fatal case of a female caused by GAS-TSS in the third trimester of pregnancy in Guangzhou, China.

Case presentation: The patient is a 33-year-old female who presented at 37 weeks' gestation with a history of three hours fever. The patient underwent an early onset and rapid progression with dramatic clinical picture and laboratory characters within 24 hours. The neonate survived after an aggressive antiinfection treatment.The GAS strains were isolated from two bottles of blood cultures and airway secretion culture, which confirmed as Streptococcus pyogenes associated with genotype emm 1 by molecular analysis.

Conclusion: Dramatic clinical picture and laboratory characters of the pregnant woman presented here might help improve clinicians' awareness and recognition of Streptococcus pyogenes, which could be of great importance for the early diagnosis of GAS- TSS in pregnancy.

\section{Background}

Streptococcus pyogenes, the causative agent of Group A streptococcal toxic shock syndrome (GAS-TSS), can cause sepsis and multi-organ failure for pregnant woman with high fatality and high fetal mortality rate [1-5]. Exposure to children (common GAS carriers) is a common way of GAS infection in pregnant women [6]. According to the literature, exotoxin A caused by type M1 or M3 of the organism was associated with invasive and toxic disease [7]. In terms of treatment, clindamycin in combination with penicillin is still the first choice for it can suppresses bacterial toxins and inhibits synthesis of $\mathrm{M}$ protein [8-9]. Besides, intravenous immunoglobulin is a better adjucant therapy [2]. Nevertheless, most pregnant women with GAS-TSS reported don't get a corrected diagnosis timely due to various factor e.g., confused clinical manifestations, lack of rapid identification and recognition for the pathogen etc. Herein, we report a case of a female who developed GAS -TSS with type emm 1 in the third trimester with dramatic clinical manifestations and characteristic laboratory findings.

\section{Case Report}

On December 29, 2017, a 33-year-old pregnant woman, gravida 1, at 37 weeks of gestation, was admitted to the emergency department of Guangdong Women and Children Hospital (Guangzhou, China) because of fever lasting for 3 hours and fetal movement increased. No abnormality was found in her regular antenatal examination. She worked as a professional teacher and had a history of exposure to fever children while there was a scarlet fever outbreak period. A week before admission, the patient had started to experience fatigue and throat discomfort.

On admission, the physical findings were within normal levels, except for a febrile condition (temperature: $39.5^{\circ} \mathrm{C}$ ). However, during the following 2 hours, an emergency cesarean section (CS) was performed due 
to fetal distress. The operation went well and the neonate was transferred to the neonatal intensive critical care unit (NICU) empirically treated with penicillin and cephalosporin for the fever. The blood culture for the infant was negative. The clinic findings demonstrated that the infant became in good health later.

Following the cesarean section, the vital signs of the patient were within normal range at first except for the poor uterine contraction with the pressing to bleed $300 \mathrm{ml}$. Then, balloon tamponade, uterine artery embolization and abdominal subtotal hysterectomy were successively performed to control the ongoing uterine bleeding. Transfusion of red cell suspension liquid, fresh frozen plasma (FFP), cryoprecipitate and apheresis platelet which were totally up to about $13,400 \mathrm{ml}$ were also initiated. The drastic deterioration of her clinical findings was shown in the Figure 2. Although the patient was received aggressive modern management with antibiotics and supportive therapy, in an attempt to correct blood clotting, promote contractions, fluid replacement, maintain water and electrolyte balance, etc., the condition deteriorated rapidly including hypotension, progressive decrease in blood oxygen saturation, systemic edema, and metabolic acidosis and the pregnant woman was finally died of septic shock and multi-organ failure within $24 \mathrm{~h}$ after admission.

The pathogenic analysis demonstrated two sets of blood culture collected on admission and after the cesarean section respectively were both present positive and Gram-positive coccus were both observed under light microscopy. After sub-culturing on $5 \%$ sheep blood agar plate under $5 \% \mathrm{CO}_{2}$ atmosphere for $24 \mathrm{~h}$, the isolate was identified as Streptococcus pyogenes using a Microflex matrix-assisted laser desorption/ionization time-of-flight mass spectrometry device (Bruker). Further, pairwise comparison of the 16S rRNA gene sequences showed that the isolate designed as SFY-1 shared highest similarities to Streptococcus pyogenes JCM $5674^{\top}$ (99.5\%) but less than $97.7 \%$ with other members of the genus Streptococcus. To determination its epidemiological characteristics, M protein gene sequence for the strain SFY-1 was detected as M1T1 genotype, which associated with toxic disease. The GenBank accession numbers for $16 \mathrm{~S}$ rRNA gene sequence and M protein gene sequence of the strain SFY-1 was MW425601 and MW699016, respectively.

In parallel, airway secretion culture was positive with colonies identified as the same as that of blood culture. Of note, vaginal and pharynx cultures were negative. Besides, antibiotic susceptibility tests for the strain SFY-1 showed susceptibility to chloramphenicol, vancomycin, trimethoprim/sulfamethoxazole, cefotaxime, benzylpenicillin, cefotaxime, amoxicillin, levofloxacin, linezolid, moxifloxacin, quinupristin/dalfopristin and rifampicin but resistance to erythromycin, clindamycin, tetracycline and telithromycin. Moreover, the lgM antibody tests for Influenza A virus was positive.

According to the clinical characteristics and microbiological results, which fulfilled criteria IA and II (A and B) of definition for GAS-TSS [10].the pregnant woman was finally diagnosed with GAS-TSS. The whole striking changing course of GAS-TSS $(e m m 1)$ and diagnosis in pregnant woman are shown in Figure 1.

\section{Discussion}


This case highlights the changes in characteristic laboratory findings of the pregnant woman with GASTSS infection caused by emm 1 in the third trimester of pregnancy, such as rapid elevated PCT, sharp drop in PLT and HBG, coagulation disorders and so on (Figure1 and 2). It is remarkable that PCT is a diagnostic marker for severe bacterial infections and sepsis and has a good relationship with the severity of sepsis. [12]. Additionally, we further confirmed the isolate by 16S rRNA gene analysis, and determined its emm type by molecular typing, so as to provide a basis for epidemiological analysis of GAS-TSS in pregnant women around the world.

In our study, although Rapid MALDI-TOF analysis was used to quickly identify the pathogenic bacteria and automated blood culture alarm system was also conducted to remind on-duty person to deal with positive specimens in time, the condition of the pregnant woman still deteriorated rapidly. The possible main reasons are as below: Firstly, when the patient appears non-specific symptoms, for example, fu-like symptoms, which may lead to missing the best treatment time; additionally, clinicians and laboratory physicians in our countries are often not familiar with this rare invasive infection caused by GAS, perhaps due to the limited number of case reports published in Chinese and English; What's worse, genotype emm1, which is often involved in STSS, was detected from GAS of the pregnant woman and of note, the isolate was resistant with clindamycin which also indicated the severity and complexity of this case.

In summary, physicians need to be aware of the characteristic laboratory findings and clinical manifestations of rare and serious infections in pregnant women caused by Streptococcus pyogene. The continuous increase of PCT and related exposure history can be used as the key points for highly suspected GAS-TSS infection.

\section{Abbreviations}

GAS-TSS, Group A streptococcal toxic shock syndrome; PCT, procalcitonin; PLT, platelet; HBG, hemoglobin; APTT, activated partial thromboplastin time; PT, prothrombin time; FDP, fibrinogen degradation product.

\section{Declarations}

\section{Ethics approval and consent to participate}

This research was carried out according to the principles of the Declaration of Helsinki and was approved by the Ethics Committees of the National Institute for Communicable Disease Control and Prevention and the Chinese Center for Disease Control and Prevention $₫$ No.202101173】.

\section{Consent for publication}

Parental written informed consent to publish this information was obtained from study participants.

\section{Availability of data and materials}


All data generated and analyzed during the study were included in the

published article.

\section{Competing Interests}

The authors declare that there are no conflicts of interest.

\section{Funding}

No funding to be declared.

\section{Authors' contributions}

L-YS performed the isolation and identification of the pathogen, Z-ML performed molecular analysis of the pathogen; C-YY and G-CM collected the case clinical data; M-XP drafted the manuscript and participated in the design of the study. L-LJ critically reviewed the manuscript. All the authors read and approved the final manuscript.

\section{Acknowledgements}

We would like to acknowledge all staff of Guangdong Women and Children Hospital.

\section{References}

1. Yamada K, Fukuda T, Kimura M et al (2012) Group A streptococcus-induced toxic shock syndrome in pregnancy: a case report of cesarean section. Masui 61:1380-1385.

2. Crum NF, Chun HM, Gaylord TG et al (2002) Group A streptococcal toxic shock syndrome developing in the third trimester of pregnancy. Infect Dis Obstet Gynecol 10:209-216.

3. Irani M, McLaren R Jr, Savel RH et al (2017) Streptococcal toxic shock syndrome occurring in the third trimester of pregnancy: A case report. J Obstet Gynaecol Res 43:1639-1643.

4. Hasegawa J, Sekizawa A, Yoshimatsu J et al (2015) Cases of death due to serious group A streptococcal toxic shock syndrome in pregnant females in Japan. Arch Gynecol Obstet 291:5-7.

5. Sugiyama T, Kobayashi T, Nagao K et al (2010) Group A streptococcal toxic shock syndrome with extremely aggressive course in the third trimester. J Obstet Gynaecol Res 36:852-855.

7. Kotb M, Norrby-Teglund A, McGeer A et al (2002) An immunogenetic and molecular basis for differences in outcomes of invasive group A streptococcal infections. Nat Med 8:1398-404.

8. Gustafson LW, Blaakær J, Helmig RB (2017) Group A streptococci infection. A systematic clinical review exemplified by cases from an obstetric department. Eur J Obstet Gynecol Reprod Biol 215:33-40. 
9. Stevens DL (1995) Streptococcal toxic-shock syndrome: spectrum of disease, pathogenesis, and new concepts in treatment. Emerg Infect Dis 1:69-78.

10. Breiman RF, Davis JP, Facklam RR et al (1993) Defining the Group A Streptococcal Toxic Shock Syndrome: Rationale and Consensus Definition. JAMA 269:390-391.

11. Oberhoffer M, Karzai W, Meier-Hellmann A et al (1998) Procalcitonin. A new diagnostic parameter for severe infections and sepsis. Anaesthesist 47:581-587.

\section{Figures}

History of fever lasting for 3 hours at 37 weeks' of gestation.

1. Fever $\left(39.5^{\circ} \mathrm{C}\right)$ and fetal movement increased \& visited our hospital

\section{3. an emergency cesarean section (CS) was performed} due to fetal distress

6.Significant increase of PCT;

Elevation of APTT, PT,FDP,D-Dimer;

Positive for plasma protamine paracoagulation test)

Dramatical drop of PLT;

Slight drop of RBC and HGB

\begin{tabular}{|c|}
\hline $\begin{array}{c}\text { 8.blood culture became positive and revealed } \\
\text { Gram-positive coccus }\end{array}$ \\
\hline
\end{tabular}

10. Edema, hyperkalemia, and metabolic acidosis and finally died of DIC

12. Streptococcus pyogenes was identified by MALDI-TOF and 16S rRNA gene analysis
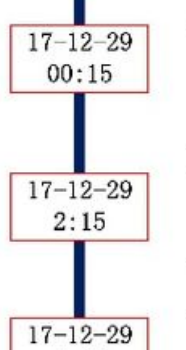

1:06

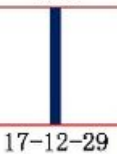

$17-12-29$

$5: 28$

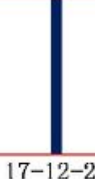

$17-12-29$

$15: 35$

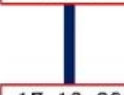

$17-12-29$

21:35

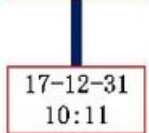

2. Physical examination and laboratory detection;

Blood,vaginal and pharynx cultures were conducted

4. the neonate was high fever and was treated with penicillin and cephalosporin.

5.The uterine contraction was poor ;

Balloon tamponade, Uterine artery

embolizationtotal \& hysterectomywas conducted;

7.Transfusion of red cell suspension liquid, fresh frodzen plasma (FFP), cryoprecipitate and apheresis platelet (totally $113,400 \mathrm{ml}$ )

9. Anti-infective therapy was switched to vancomycin and ornidazole

11. Blood culture performed after CS were the same as before

13. serotype M1 for the isolation was conformed by sequencing

The pregnant woman was diagnosed as streptococcal toxic shock syndrome;

The neonate became in good health after an timely anti-infective management

Figure 1

Course of disease and maternal and infant outcome of pregnant women infected with GAS-TSS. 


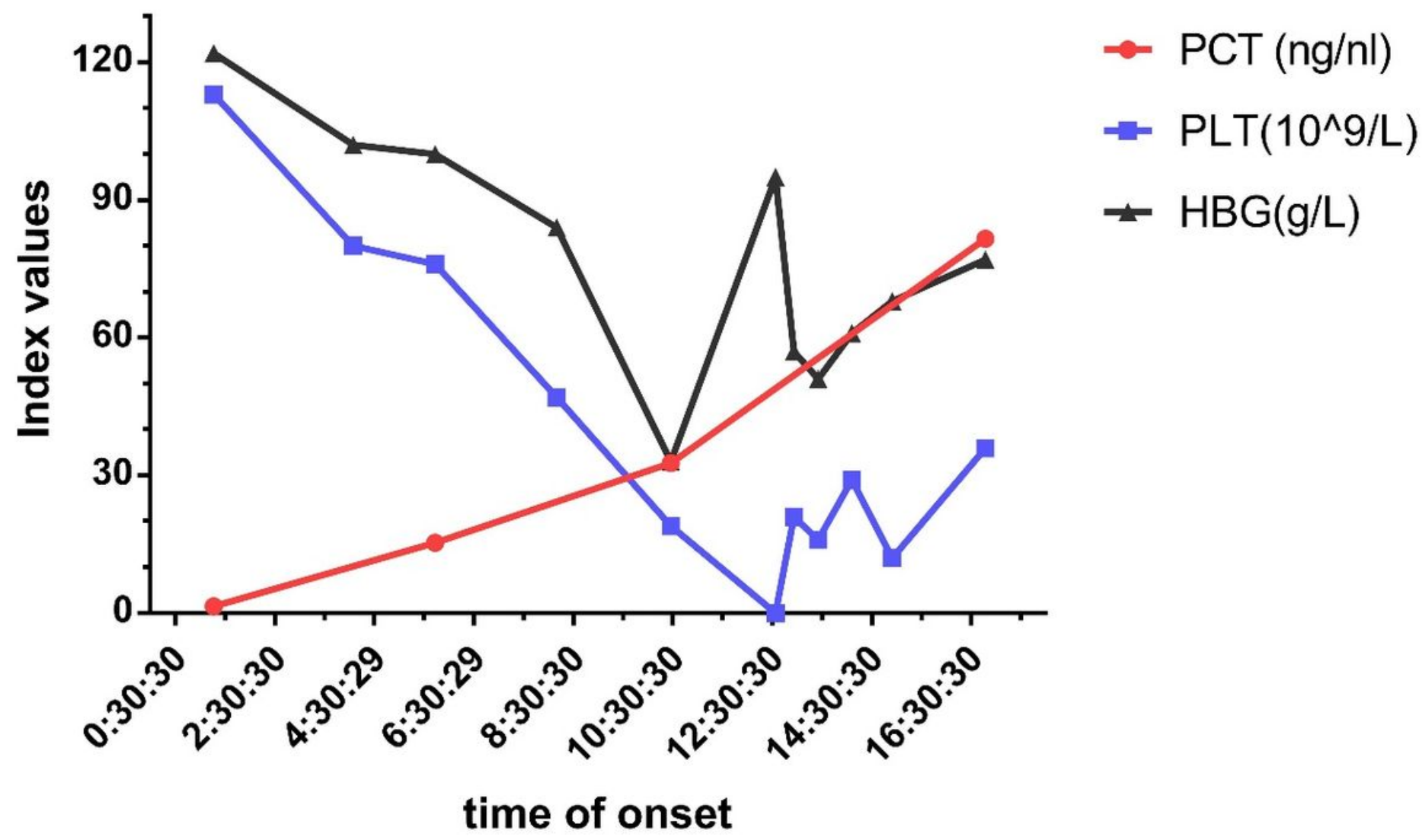

Figure 2

The change trend of PCT $\triangle \mathrm{PLT}$ and HBG values of pregnant woman infected with GAS-TSS during the whole course of disease. 